\title{
Manifestaciones clínicas retinianas durante el puerperio inmediato en pacientes con preeclampsia en un hospital de México
}

\section{Clinical retinal findings during the immediate postpartum period in patients with preeclampsia in a Mexican hospital}

\author{
Geovanni J. Ríos-Nequis, Héctor G. Flores-Ceballos*, Adriana Saucedo-Castillo, \\ Arthur Levine-Berebichez, Benito Celis-Suazo, Sergio Rojas-Juárez y Juan A. Ramírez-Estudillo \\ Departamento de Retina y Vítreo, Fundación Hospital Nuestra Señora de la Luz, Ciudad de México, México
}

\begin{abstract}
Resumen
Introducción y objetivo: Los trastornos hipertensivos durante la gestación son algunas de las principales complicaciones obstétricas en muchos países del mundo. En la preeclampsia, las pacientes pueden percibir fenómenos ópticos que pueden predecir la evolución de preeclampsia a eclampsia, ya que los cambios oftalmológicos se manifiestan como una retinopatía hipertensiva. El objetivo de este estudio es describir los hallazgos clínicos retinianos, mediante oftalmoscopia indirecta, en pacientes con preeclampsia durante el puerperio inmediato. Material y métodos: Estudio descriptivo, observacional, prospectivo y transversal, realizado de agosto a diciembre de 2014, en pacientes con preeclampsia hospitalizadas en un hospital materno-infantil de la Ciudad de México durante el puerperio inmediato, a quienes se les realizó una exploración oftalmológica mediante oftalmoscopia indirecta. Se revisó el fondo de ojo y se describieron los hallazgos clínicos y la clasificación de retinopatía hipertensiva en caso de existir. Así mismo, se identificó la relación de factores que puedan influir en el grado de retinopatía hipertensiva. Resultados: Se valoraron 26 pacientes con preeclampsia severa, 5 pacientes con preeclampsia leve y 1 paciente con síndrome de HELLP incompleto, de un total de 32 pacientes incluidas en el estudio. Los signos clínicos identificados con mayor frecuencia fueron vasoespasmo y la presencia de cruces arteriovenosos. El $34.61 \%$ de pacientes diagnosticadas con preeclampsia severa presentaron retinopatía hipertensiva grado II. La relación del grado de retinopatía hipertensiva con la edad tuvo una significancia estadística de $p=0.044$. Conclusiones: Diferentes grados de retinopatía hipertensiva (grado I, II y III) fueron observados en pacientes con preeclampsia severa. No se encontraron pacientes con neuropatía hipertensiva o con datos de isquemia coroidea asociados a desprendimiento de retina seroso. La edad fue el factor con mayor asociación al grado de retinopatía hipertensiva.
\end{abstract}

Palabras clave: Preeclampsia. Retinopatía hipertensiva. Vasoespasmo. Isquemia retiniana.

\section{Abstract}

Introduction and objective: Hypertensive disorders during pregnancy are one of the main obstetric complications in many countries. In preeclampsia, patients may perceive optical phenomena that can predict the evolution from preeclampsia to eclampsia, because ophthalmic changes manifest as a hypertensive retinopathy. The aim of this study is to describe the

Correspondencia:

*Héctor G. Flores-Ceballos

Ezequiel Montes, 135

Col. Tabacalera, Del. Cuauhtémoc

Fecha de recepción: 01-07-2019

Fecha de aceptación: 09-01-2020

E-mail: hectorgabrielf@gmail.com

DOI: 10.24875/RMO.M20000115
Disponible en internet: 01-05-2020 Rev Mex Oftalmol. 2020;94(3):119-123

www.rmo.com.mx 0187-4519/๑ 2020 Sociedad Mexicana de Oftalmología. Publicado por Permanyer. Este es un artículo open access bajo la licencia CC BY-NC-ND (http://creativecommons.org/licenses/by-nc-nd/4.0/). 
clinical retinal findings in patients diagnosed with preeclampsia in the immediate postpartum by indirect ophthalmoscopy. Methods: Descriptive, observational, prospective and cross-sectional study performed from August to December 2014, in patients diagnosed with preeclampsia admitted to a mother and child hospital, in the immediate postpartum, who underwent ophthalmic examination by indirect ophthalmoscopy. Fundus eye examination was performed describing clinical findings, and hypertensive retinopathy grading was done if present. Also, we evaluated a possible correlation between different factors that may influence the degree of hypertensive retinopathy. Results: Twenty-six patients with severe preeclampsia, 5 patients with mild preeclampsia and 1 patient with incomplete HELLP syndrome were examined, with a total of 32 patients enrolled. The prevalent clinical signs observed were vasospasm and arteriovenous crossings. We observed grade II hypertensive retinopathy in $34.61 \%$ of patients diagnosed with severe preeclampsia. Age was the main factor correlated with the degree of hypertensive retinopathy, with statistical significance $(p=0.044)$. Conclusions: Different grades of hypertensive retinopathy (grade I, II and III) were observed in patients with severe preeclampsia. No patients showed hypertensive neuropathy or choroidal ischemia associated with serous retinal detachment. Age is the main factor associated with the severity of hypertensive retinopathy.

Key words: Preeclampsia. Hypertensive Retinopathy. Vasospasm. Retinal Ischemia.

\section{Introducción}

Los trastornos hipertensivos durante la gestación son algunas de las principales complicaciones obstétricas en muchos países del mundo, y constituyen una de las primeras causas de morbilidad y mortalidad materna, fetal y neonatal. La frecuencia reportada muestra gran variabilidad y sus valores oscilan del 12 al $22 \%$. México presenta cifras semejantes. La preeclampsia/eclampsia origina el $70 \%$ de los estados hipertensivos durante el embarazo, y el $30 \%$ lo representan pacientes con hipertensión crónica preexistente.

En el año 2000 el Sistema Nacional de Salud de México reportó 466 defunciones por trastornos hipertensivos durante la gestación, lo que representa un $35.17 \%$ de las causas de mortalidad materna1.

Esta descrita la importancia de una adecuada exploración del fondo de ojo y la información valiosa que esta puede proporcionarnos. Durante el embarazo existen cambios oculares fisiológicos, como hipotensión ocular mediada por un aumento en la salida de humor acuoso ${ }^{2}$, disminución de la sensibilidad corneal con probable edema ${ }^{3}$, cambios patológicos que pueden resultar en el desarrollo de coriorretinopatía serosa central ${ }^{4}$, crecimiento de tumoraciones uveales ${ }^{5}$ o progresión de retinopatía diabética ${ }^{6}$. En la preeclampsia, las pacientes pueden percibir fenómenos ópticos que pueden predecir la evolución de preeclampsia a eclampsia, ya que los cambios oftalmológicos se manifiestan como una retinopatía hipertensiva. El signo clínico más frecuente son los espasmos arteriolares, que se reportan en un 40 a $100 \%$ de pacientes con preeclampsia y se acompañan de zonas de isquemia retiniana ${ }^{7}$. Se pueden presentar hemorragias, exudados, edema retiniano difuso y papiledema. Se reportan desprendimientos de retina serosos en el $10 \%$ de pacientes con eclampsia y el 1 a $2 \%$ de pacientes con preeclampsia severa ${ }^{8}$.

El examen oftalmoscópico nos puede ayudar a estimar el estado vascular de la placenta y predecir el aumento de la severidad de la toxemia relacionado con los hallazgos oculares.

Dentro de la fisiopatología de la preeclampsia, se encuentra una pérdida del balance de factores angiogénicos, tales como factor de crecimiento vascular endotelial y factor de crecimiento derivado de plaquetas. Estos factores se encuentran sobreexpresados durante el embarazo y su inhibición produce hipertensión y proteinuria dependiente de la dosis ${ }^{9}$.

Se han realizado estudios donde se evalúa la severidad de la retinopatía hipertensiva con el grado de severidad en la alteración sistémica por preeclampsia, y se ha encontrado una correlación directa ${ }^{10}$.

El objetivo de este estudio fue describir los hallazgos encontrados en el examen de fondo de ojo durante el puerperio inmediato, en pacientes que previamente habían sido diagnosticadas con preeclampsia. Esto con la finalidad de evaluar y comparar los hallazgos de este estudio con aquellos descritos en la literatura en poblaciones distintas a la mexicana, y de esta forma establecer la importancia de la exploración oftalmológica en pacientes con preeclampsia.

\section{Material y métodos}

Este fue un estudio descriptivo, observacional, prospectivo y transversal que se realizó de agosto de 2014 a diciembre 2014. Se revisaron pacientes de sexo femenino hospitalizadas por diagnóstico de preeclampsia en un hospital maternoinfantil de la Ciudad de 
México durante el puerperio inmediato. En caso de presentar preeclampsia severa, se revisaron en el servicio de terapia intensiva; y en el caso de presentar preeclampsia leve, se revisaron en el servicio de puerperio patológico.

Se obtuvo la firma de consentimientos informados de las pacientes para poder realizar la revisión y recolección de datos para la realización del estudio. Todos los procedimientos se llevaron a cabo de acuerdo con los estándares éticos del comité de investigación institucional de la Fundación Hospital Nuestra Señora de la Luz y cumpliendo los principios éticos de la Declaración de Helsinki.

A las pacientes se les realizó un estudio de fondo de ojo mediante oftalmoscopio indirecto (Vantage Plus LED, Keeler, Opthalmic Instruments, Reino Unido) bajo midriasis farmacológica con tropicamida/fenilefrina (T-P Ofteno, Laboratorios Sophia, México) y lente aérea de 20 D (Volk Optical, Inc., EE.UU), utilizando la mínima iluminación y realizando oclusión de puntos lagrimales para minimizar la absorción sistémica del colirio. No hubo eventos adversos relacionados a la aplicación del colirio o a la revisión oftalmológica.

Se estadificó el grado de retinopatía hipertensiva utilizando la clasificación de Keith-Wagener-Barker $(K W B)^{11}$. Se tomaron registros de resultados de laboratorio y de signos vitales del expediente clínico de las pacientes, así como el registro del tratamiento antihipertensivo instituido.

La clasificación de la preeclampsia se realizó de acuerdo a los lineamientos propuestos por el Colegio Americano de Obstetras y Ginecólogos ${ }^{12}$.

Para el análisis estadístico se realizó un modelo de regresión lineal para relacionar el grado de retinopatía hipertensiva con los niveles de enzimas hepáticas, el conteo plaquetario, la tensión arterial media, la proteinuria, la presencia de edema de miembros inferiores, la edad y el tratamiento antihipertensivo. Se utilizó el programa SPSS, Nueva York, EE.UU. y se consideró un valor de $p \leq 0.05$ como estadísticamente significativo.

\section{Resultados}

Se estudiaron 32 pacientes de sexo femenino con una edad media de 23.84 ( $D E \pm 5.7$ ) en el puerperio inmediato de un hospital maternoinfantil de la Ciudad de México (Tabla 1).

De acuerdo al grado de preeclampsia, se obtuvieron tres grupos: 1) preeclampsia severa, $6(23.07 \%)$ pacientes presentaron retinopatía hipertensiva grado III, $9(34.61 \%)$ presentaron grado II, 5 (19.23\%) presentaron
Tabla 1. Datos demográficos

\begin{tabular}{|l|c|}
\hline Número de pacientes & 32 \\
\hline Edad promedio (DE) & $23.8( \pm 5.7)$ \\
\hline Preeclampsia leve, $\mathrm{n}(\%)$ & $5(15.6 \%)$ \\
\hline Preeclampsia severa, $\mathrm{n}(\%)$ & $26(81.2 \%)$ \\
\hline Síndrome de HELLP incompleto, $\mathrm{n}(\%)$ & $1(3.1 \%)$ \\
\hline Síntomas visuales (fosfenos), $\mathrm{n}(\%)$ & $2(6.2 \%)$ \\
\hline Tensión arterial media & 104.04 \\
\hline
\end{tabular}

grado I y $6(23.07 \%)$ presentaron grado 0; 2 . preeclampsia leve, $2(40 \%)$ pacientes presentaron retinopatía hipertensiva grado II, 1 (20\%) presentó grado I y $2(40 \%)$ presentaron grado 0 ; y 3) síndrome de HELLP incompleto, $1(100 \%)$ paciente presentó retinopatía hipertensiva grado I.

En las pacientes con preeclampsia severa, predominaron el vasoespasmo (80.76\%) y los cruces arteriovenosos $(57.69 \%)$ como signos clínicos. Las pacientes con preeclampsia leve tuvieron resultados similares, el $40 \%$ presentaron cruces arteriovenosos y el $60 \%$ vasoespasmo. La paciente con síndrome de HELLP incompleto presentó vasoespasmo (100\%) (Tabla 2).

Durante la exploración de las pacientes, se identificaron 16 con presencia de edema de miembros inferiores, las principales manifestaciones clínicas fueron cruces arteriovenosos (56.2\%) y vasoespasmo (68.7\%); y 14 pacientes presentaron proteinuria, de las cuales, la mayoría tenían como signo clínico predominante vasoespasmo $(85.7 \%)$, seguido de cruces arteriovenosos $(71.42 \%)$. Las enzimas hepáticas se identificaron con niveles alterados en 10 pacientes, de las cuales, el hallazgo clínico oftalmológico más frecuente fue el vasoespasmo (80\%) (Tabla 3).

Las pacientes con preeclampsia y edema de miembros inferiores presentaron con mayor frecuencia datos clínicos compatibles con retinopatía hipertensiva grado II y III de KWB. En las pacientes con proteinuria, los grados II y III estuvieron presentes hasta en el $70 \%$ de las pacientes, mientras que en las pacientes con enzimas hepáticas alteradas, se identificó retinopatía hipertensiva grado I predominantemente (40\%) (Tabla 4).

El $46.87 \%$ de las pacientes se encontraba bajo tratamiento con nifedipino, mientras que el $15.62 \%$ recibió tratamiento combinado con propanolol y nifedipino y el $37.5 \%$ no requirió tratamiento hipotensor, lo que resultó en una tensión arterial media de 104.04 entre los tres grupos. 
Tabla 2. Signos clínicos de acuerdo a la severidad de preeclampsia

\begin{tabular}{|c|c|c|c|c|c|}
\hline & $\begin{array}{l}\text { Hemorragias } \\
\text { intrarretinianas }\end{array}$ & $\begin{array}{c}\text { Exudados } \\
\text { blandos }\end{array}$ & $\begin{array}{c}\text { Cruces } \\
\text { arteriovenosos }\end{array}$ & Vasoespasmo & $\begin{array}{l}\text { Tortuosidad } \\
\text { vascular }\end{array}$ \\
\hline & $\mathbf{N}(\%)$ & $\mathbf{N}(\%)$ & $\mathbf{N}(\%)$ & $\mathbf{N}(\%)$ & $\mathbf{N}(\%)$ \\
\hline Preeclampsia leve & $0(0)$ & $0(0)$ & $2(40)$ & $3(60)$ & $0(0)$ \\
\hline Preeclampsia severa & $3(11.5)$ & $5(19.2)$ & $15(57.6)$ & $21(80.7)$ & $3(11.5)$ \\
\hline Síndrome HELLP incompleto & $0(10)$ & $0(0)$ & $0(0)$ & $1(100)$ & $0(0)$ \\
\hline
\end{tabular}

Tabla 3. Signos clínicos presentados en pacientes con edema de miembros inferiores, proteinuria y alteración enzimas hepáticas

\begin{tabular}{|l|c|c|c|cc|c|}
\hline & $\begin{array}{c}\text { Hemorragias } \\
\text { intrarretinianas }\end{array}$ & $\begin{array}{c}\text { Exudados } \\
\text { blandos }\end{array}$ & $\begin{array}{c}\text { Cruces } \\
\text { arteriovenosos }\end{array}$ & $\begin{array}{c}\text { Vasoespasmo } \\
\text { Tortuosidad } \\
\text { vascular }\end{array}$ \\
\cline { 2 - 6 } & $N(\%)$ & $N(\%)$ & $N(\%)$ & $N(\%)$ & $N(\%)$ \\
\hline Edema de miembros inferiores & $1(6.2)$ & $3(18.7)$ & $9(56.2)$ & $11(68.7)$ & $1(6.2)$ \\
\hline Proteinuria & $1(7.14)$ & $3(21.42)$ & $10(71.42)$ & $12(85.7)$ & $2(14.28)$ \\
\hline Alteración enzimas hepáticas & $1(10)$ & $2(20)$ & $4(40)$ & $8(80)$ & $1(10)$
\end{tabular}

Tabla 4. Grado de retinopatía hipertensiva en pacientes con edema de miembros inferiores, proteinuria y alteración de las enzimas hepáticas

\begin{tabular}{|l|c|c|c|}
\hline $\begin{array}{l}\text { Clasificación } \\
\text { KWB }\end{array}$ & $\begin{array}{r}\text { Edema de } \\
\text { miembros } \\
\text { inferiores }\end{array}$ & Proteinuria & $\begin{array}{c}\text { Alteración de } \\
\text { las enzimas } \\
\text { hepáticas }\end{array}$ \\
\hline N (\%) & N $(\%)$ & N $(\%)$ \\
\hline IV & $0(0)$ & $0(0)$ & $0(0)$ \\
\hline III & $4(25)$ & $4(28.5)$ & $2(20)$ \\
\hline II & $5(31.2)$ & $6(42.8)$ & $2(20)$ \\
\hline I & $2(12.5)$ & $2(14.2)$ & $4(40)$ \\
\hline 0 & $5(31.2)$ & $2(14.2)$ & $2(20)$ \\
\hline
\end{tabular}

KWB: Keith-Wagener-Barker.

La comparación entre el grado de retinopatía hipertensiva y la edad fue estadísticamente significativa $(p=0.044)$, no así al realizar la comparación con las enzimas hepáticas, proteinuria, edema de miembros inferiores, la tensión arterial media y el conteo plaquetario (Tabla 5).

\section{Discusión}

El hallazgo fundoscópico más frecuente fueron los cruces arteriovenosos y el vasoespasmo arteriolar en los tres grupos reportados.
Tabla 5. Modelo de regresión lineal comparando el grado de retinopatía hipertensiva con la edad, enzimas hepáticas, tensión arterial media, proteinuria, trombocitopenia y edema de miembros inferiores

\begin{tabular}{|l|c|c|}
\hline Grado de retinopatía hipertensiva vs. & Valor F & Significancia \\
\hline Edad & 4.424 & 0.044 \\
AST & 0.118 & 0.734 \\
\hline ALT & 0.001 & 0.972 \\
\hline Tensión arterial media & 0.499 & 0.485 \\
\hline Proteinuria & 1.657 & 0.208 \\
\hline Trombocitopenia & 0.219 & 0.643 \\
\hline Edema de miembros inferiores & 0.375 & 0.545
\end{tabular}

ALT: alanino aminotransferasa; ASL: aspartato aminotransferasa.

En todos los grupos, el hallazgo más frecuente es el vasoespasmo generalizado, sin embargo, son hallazgos diferentes a los publicados por Rasdi, et. al, quienes reportan que hasta el $70 \%$ de las pacientes tenían un fondo de ojo sin alteraciones y sin retinopatía hipertensiva hasta en el $82 \%$, pero no hacen la distinción de grupos de acuerdo a la severidad de la preeclampsia. En su estudio reportaron 1 paciente con desprendimiento de retina seroso bilateral, mientras que en nuestro estudio ninguna paciente presentó desprendimiento de retina seroso ${ }^{12}$. 
Tadin, et al, demuestran una correlación importante entre el grado de hipertensión y la severidad de la retinopatía hipertensiva, en contraste con nuestro estudio, donde no es constante este hallazgo, sin embargo, el grupo de pacientes estudiado por Tadin eran aún gestantes ${ }^{10}$.

Existe un mayor grado y proporción de retinopatía hipertensiva en pacientes con preeclampsia severa que en pacientes con preeclampsia leve.

El grupo con mayor grado de severidad de la retinopatía hipertensiva fue el grupo asociado a proteinuria, seguido del grupo asociado a edema de miembros inferiores.

Los resultados de los hallazgos fundoscópicos son homogéneos en todos los grupos.

Solamente se presentaron síntomas visuales, tales como fosfenos en 2 pacientes $(6.2 \%)$, sin reportes de metamorfopsia, micropsia o alteraciones de la percepción del color.

En los pacientes estudiados no se encontraron pacientes que tuviesen retinopatía hipertensiva grado IV, tampoco desprendimientos de retina serosos. La edad fue el factor que tuvo mayor relación al grado de retinopatía hipertensiva, tal vez explicándose por el desarrollo progresivo de ateroesclerosis, que justificaría los hallazgos clínicos descritos a pesar de encontrarse cifras tensionales con tendencia a la normalidad.

Dentro de las limitaciones del estudio están la necesidad de una muestra más amplia para validar los hallazgos descritos en el mismo y la necesidad de un grupo control para realizar un estudio comparativo con población sana. Así mismo, otra limitante fue que no se tuvo el registro de la presión arterial previa a la instauración del tratamiento antihipertensivo para de esta forma poder tratar de identificar si existían cifras tensionales mayores y su relación con el grado de retinopatía hipertensiva.

\section{Conclusiones}

Mediante el estudio realizado se describen los hallazgos fundoscópicos en pacientes con preeclampsia de un hospital en México. Se observó retinopatía hipertensiva grado I, II y III en las pacientes con preeclampsia severa, mientras que en pacientes con preeclampsia leve se observó retinopatía hipertensiva grado I y II. La edad es un factor importante que se asocia con el grado de daño microvascular asociado a hipertensión arterial y, por lo tanto, las manifestaciones oftalmológicas que se puedan identificar.

\section{Financiamiento}

El presente trabajo fue financiado por el patronato de la Fundación Hospital Nuestra Señora de la Luz, IAP. La organización de financiación no tuvo ningún rol en el diseño o conducta de la presente investigación.

\section{Conflicto de intereses}

Los autores declaran no tener ningún conflicto de intereses.

\section{Responsabilidades éticas}

Protección de personas y animales. Los autores declaran que los procedimientos seguidos se conformaron a las normas éticas del comité de experimentación humana responsable y de acuerdo con la Asociación Médica Mundial y la Declaración de Helsinki.

Confidencialidad de los datos. Los autores declaran que han seguido los protocolos de su centro de trabajo sobre la publicación de datos de pacientes.

Derecho a la privacidad y consentimiento informado. Los autores han obtenido el consentimiento informado de los pacientes y/o sujetos referidos en el artículo. Este documento obra en poder del autor de correspondencia.

\section{Bibliografía}

1. Prevención y manejo de la preeclampsia / Eclampsia Lineamiento Técnico, 2007 SSA. [Consultada el 6 de mayo de 2014]. Disponible en: http://www. salud.gob.mx/unidades/cdi/documentos/PREECLAMPSIA_ECLAMPSIA_ lin-2007.pdf. Phillips C, Gore S. Ocular hypotensive effect of late pregnancy with and without high blood pressure. Br J Ophthalmol. 1985;69(2):117-9.

2. Millodot $\mathrm{M}$. The influence of pregnancy on the sensitivity of the cornea. $\mathrm{Br}$ J Ophthalmol. 1977;61(10):646-9.

3. Fastenberg D, Ober R. Central Serous Choroidopathy in Pregnancy. Archives of Ophthalmology. 1983;101(7):1055-8.

4. Seddon J, MacLaughlin D, Albert D, Gragoudas E, Ference M. Uveal melanomas presenting during pregnancy and the investigation of oestrogen receptors in melanomas. Br J Ophthalmol. 1982;66(11):695-704.

5. Sunness J. The pregnant woman's eye. Surv Ophthalmol. 1988;32(4):219-38.

6. Wagener $\mathrm{H}$. Arterioles of The Retina in Toxemia of Pregnancy. J Am Med Assoc. 1933;101(18):1380.

7. Fry W. Extensive Bilateral Retinal Detachment in Eclampsia, with Complete Reattachment. Arch of Ophthalmol. 1929;1(5):609.

8. Ostendorf T, De Vriese A, Floege J. Renal side effects of anti-VEGF therapy in man: a new test system. Nephrol Dial Transplant. 2007;22(10):2778-80.

9. Tadin I, Bojić L, Mimica M, Karelović D, Dogas Z. Hypertensive Retinopathy and Preeclampsia. Coll Antropol. 2001:25(1):77- 81.

10. Walsh J. Hypertensive Retinopathy. Ophthalmology. 1982;89(10):1127-131.

11. ACOG Practice Bulletin No. 202. Obstet Gynecol. 2019;133(1):e1-e25.

12. Rasdi AR, Nik-Ahmad-Zuky NL, Bakiah S, Shatriah I. Hypertensive Retinopathy and visual outcome in Hypertensive Disorders in Pregnancy. Med J Malaysia. 2011;66(1):42-7. 